Fonseca-Grandón, G. R., Gabalán-Coello, J., y Vásquez-Rizo, F. E. (enero-abril, 2022).

Tipologías de estudiantes que permanecen y abandonan la Universidad: aproximación comprensiva desde el currículum y el proyecto de vida. Revista Virtual Universidad Católica del Norte, (65), 5-30. https://www.doi.org/10.35575/rvucn.n65a2

\title{
Tipologías de estudiantes que permanecen y abandonan la Universidad: aproximación comprensiva desde el currículum y el proyecto de vida
}

Typologies of students who remain and leave the University: comprehensive approach from the curriculum and life project

\section{Gonzalo Raúl Fonseca-Grandón}

$\mathrm{PhD}$ en Educación Comparada y Fundamentos de la Educación

Departamento de Ciencias de la Educación, Universidad de Concepción

Concepción, Chile

gfonseca@udec.cl

Orcid: http://orcid.org/0000-0003-1063-1319

\section{Jesús Gabalán-Coello}

$\mathrm{PhD}$ en Medición y Evaluación en Educación

Corporación Penser

Pereira, Colombia

igabalan@penser.org

Orcid: http://orcid.org/0000-0001-7674-8849

CvLAC:

http://scienti.colciencias.gov.co:8081/cvlac/visualizador/generarCurriculoCv.do?cod_rh=0000549339

\section{Fredy Eduardo Vásquez-Rizo}

$\mathrm{PhD}$ en Gestión de la Información y la Comunicación en las Organizaciones

Departamento de Comunicación, Universidad Autónoma de Occidente

Santiago de Cali, Colombia

fvasquez@uao.edu.co

Orcid: http://orcid.org/0000-0003-1398-6174

CvLAC:

http://scienti.colciencias.gov.co:8081/cvlac/visualizador/generarCurriculoCv.do?cod_rh=0000675962

Recibido: 15 de febrero de 2021

Evaluado: 30 de agosto de 2021

Aprobado: 06 de diciembre de 2021 
Tipo de artículo: Investigación Científica y Tecnológica

\title{
Resumen
}

La deserción estudiantil es uno de los temas que más inquieta a las instituciones de educación superior, razón por la cual intentan caracterizar a su población estudiantil como estrategia de prevención y acción, a partir del entendimiento de su relacionamiento con la institución e intereses de vida. En este artículo se analizaron las trayectorias formativas de estudiantes de una universidad chilena, asociadas a su decisión de permanencia o abandono, a partir de la influencia del currículum universitario y el proyecto de vida, definiendo cuatro tipologías estudiantiles. Se empleó metodología mixta, a través de técnicas cuantitativas y cualitativas (esta última incorporando análisis temático para la construcción categorial), analizando para ello dos grupos (permanecen y abandonan), lo que permitió establecer dichas tipologías a partir de una estructura relacional de factores asociados a la decisión, complementándose con opiniones estudiantiles en entrevistas semiestructuradas. En el primer caso se abordó un análisis de datos cifrados (1454 registros), y en el segundo, un cuestionario en línea aplicado a 171 estudiantes. Se concluye que las categorías analizadas (Tiempo de extrañeza, Tiempo de aprendizaje y Tiempo de afiliación) presentan una incidencia en el concepto de permanencia, vista desde la arista del currículum universitario y el proyecto de vida.

Palabras clave: Currículum; Deserción escolar; Investigación pedagógica; Planificación de la educación; Sistema educativo.

\begin{abstract}
Student desertion is one of the issues that most concerns higher education institutions, which is why they try to characterize their student population as a prevention and action strategy, based on the understanding of their relationship with the institution and life interests. In this article, the formative trajectories of students from a Chilean university were analyzed, associated with their decision to stay or drop out, based on the influence of the university curriculum and the life project, defining four student typologies. Mixed methodology was used, through quantitative and qualitative techniques (the latter incorporating thematic analysis for the categorical construction),
\end{abstract}


analyzing two groups (remain and leave), which allowed establishing these typologies from a relational structure of associated factors to the decision, supplemented with student opinions in semi-structured interviews. In the first case, an analysis of encrypted data (1454 records) was approached, and in the second, an online questionnaire applied to 171 students. It is concluded that the categories analyzed (Strangeness time, Learning time and Affiliation time) have an impact on the concept of permanence, seen from the edge of the university curriculum and the life project.

Keywords: Curriculum; Dropping out; Educational research; Educational planning; Education system.

\section{Introducción}

En los últimos 50 años la matrícula en educación superior aumentó 15 veces en el ámbito mundial (Organización de las Naciones Unidas para la Educación, la Ciencia y la Cultura [UNESCO], 2015), involucrando un cambio en la naturaleza de los sistemas educativos. En Chile, los indicadores evidencian un aumento de 50,2 \% entre el período 2008-2017, de acuerdo con las estadísticas del Servicio de Información de Educación Superior [SIES] (2018), del Ministerio de Educación. Esta situación ha permitido la incorporación de grupos socioeconómicos que históricamente han estado subrepresentados en dicho nivel educativo, fenómeno señalado por Vandelannote \& Demanet (2021).

Al margen de esta explosión de matrícula, las tasas de deserción demuestran importantes inconvenientes de los estudiantes para terminar la Universidad (Castrillón Gómez et al., 2020; Gómez-Molina et al. 2019; Wild \& Schoulze, 2020). En Chile, el SIES (2018) señala que más del $20 \%$ de los educandos que entra a la educación superior abandona sus estudios solo durante el primer año, y esto se incrementa a más del $50 \%$ en algunas áreas, durante el desarrollo formativo. Esta situación es concordante con los datos que se tienen de Latinoamérica, por ejemplo, en Colombia, donde el abandono de la educación superior en el primer año es del $47 \%$, siendo una de las más altas de la región (Asociación Colombiana de Universidades [ASCUN], 2018), lo que pone de manifiesto un fenómeno que, pese a las estrategias incorporadas, dista de estar cerca de su terminación. 
En este contexto, si bien los cambios impulsados al currículum universitario a partir del Proceso de Bolonia constituyen, desde la perspectiva institucional, una estrategia sustantiva para combatir la problemática del abandono (Rodríguez Gómez, 2018), esta ha sido una situación escasamente explorada (Christiane, 2019; European Commission, 2018), a pesar de algunos esfuerzos aislados, iniciando con Tinto (1975) en el campo de la retención estudiantil y las comunidades de aprendizaje, que han detectado la necesidad de indagar acerca de la relación existente entre la innovación pedagógica y su efecto en la permanencia estudiantil universitaria (Ñañez Silva et al., 2021; Velásquez Narváez y González Medina, 2017).

Por tanto, el presente artículo pretende contribuir a la discusión, a partir de la concepción del currículum universitario como generador de resultados (Nurie, 2019; Seah et al., 2020) y cómo sus diversas reformas en una universidad chilena impactan las decisiones de permanencia o abandono de los estudios superiores, permitiendo identificar incluso tipologías estudiantiles asociadas a esta problemática, enmarcada en lo que el estudiante considera como un aspecto relacionado a su proyecto de vida.

\section{Revisión de la literatura}

Las investigaciones sobre permanencia y deserción en estudios superiores, desarrolladas por la corriente funcionalista norteamericana, han sido ejecutadas desde la década de 1970 por un grupo importante de autores que promueven aproximaciones teórico-metodológicas cuantitativas (Bean \& Metzner, 1985; Klees, 2017; Köhler et al., 2017; Tinto, 1975), todos ellos reconocidos dentro de la perspectiva organizacional (Fonseca Grandón y García, 2016). Lo anterior, sumado a trabajos de otras perspectivas (Modena et al., 2020; Savela, 2017), destaca "los procesos de integración académica e integración social como variables explicativas de la permanencia universitaria" (Fonseca Grandón, 2018).

Adicional a esto, existe una corriente teórico-metodológica cualitativa representada principalmente por autores provenientes de la sociología francesa (Coulon, 2017; Dubet, 2018), quienes han ayudado a explicar lo que acontece con los estudiantes que se retiran de la Universidad. En este sentido, Coulon (2017) considera que la incorporación al mundo universitario es un proceso conformado por tres momentos: "tiempo de extrañeza", que revela la desvinculación 
con el estatus anterior; "tiempo de aprendizaje", asociado a la ambigüedad entre las pasadas y nuevas directrices; y “tiempo de afiliación”, estado que posibilita a los estudiantes la interpretación de las nuevas reglas y su adaptación a la Universidad.

Dicha trayectoria está mediada por la experiencia vivida por los estudiantes universitarios, desde la cual se desprenden diversas tipologías estudiantiles, siendo Dubet (2018) quien deja entrever la autonomía y disciplina personal que implica la inserción en la Universidad, lejos de las presiones familiares (aspecto considerado como trascendental para el desenvolvimiento formativo y social, según Rodríguez Bustamante et al. (2021)) y de los profesores del nivel secundario; situación que moldea, en general, los distintos tipos de estudiantes: aquellos que no consiguen estructurarse ni disciplinarse, y aquellos que se responsabilizan de sí mismos.

Estas tipologías consisten en clasificaciones que se establecen a partir de las características que comparten grupos de estudiantes, las cuales varían en su especificidad, dependiendo del autor que las establezca o analice, como es el caso de Soler Julve (2014), quien clasifica a los estudiantes en cuatro conglomerados: a) Integrados: dedicados intensamente al estudio, con un alto cumplimiento de sus responsabilidades académicas y rendimiento académico, pero con competencias sociales y comunicativas relativamente débiles; b) Desajustados: estudiantes a tiempo completo, pero sin un fuerte vínculo con el estudio, lo que se manifiesta en su rendimiento académico y en la ausencia de responsabilidades académicas; c) Trabajadores: estudiantes a tiempo parcial, que trabajan, por lo que su vinculación con el estudio es débil, al igual que su sociabilidad; y d) Vocacionales: sin obligaciones laborales, responsables, pero atentos solo a aspectos concretos de su formación, según sus intereses.

Otro ejemplo de este tipo de clasificaciones es el propuesto por Pineda Lezama y Alcántara Galdámez (2017), quienes clasifican a los estudiantes según la preferencia de sus hábitos de estudio. De esta manera, los tipifican en: a) Organizados en la planificación y seguimiento de sus horarios, b) Estructurados en su metodología de estudio, c) Meticulosos en su comportamiento frente a los exámenes, d) Resolutivos frente a los problemas de clase, e) Dependientes de su estado motivacional y de ánimo y f) Desinteresados.

En el mismo año aparece un análisis desarrollado por Hurtado Bello et al. (2017), quienes organizan a los estudiantes en: a) Reflexivos, aquellos que asisten a clase con preparación previa, participan constantemente y su colaboración es dinámica; b) Teóricos, son quienes emplean un 
lenguaje fluido, participan activamente y son muy organizados; c) Reflexivo/teóricos, aquellos que combinan los dos anteriores; y tres conglomerados antagónicos a cada uno de estos roles (No Reflexivos, no Teóricos y no Reflexivos ni Teóricos).

Por su parte, Jenert et al. (2017) clasifican a los estudiantes según su incorporación y articulación a la educación superior, tomando como referente su primer año de estudios, considerando a dicho año como un espectro fundamental para su adaptación, en el que intervienen incluso factores asociados con la salud mental y psicológica que afectan el rendimiento (Chalela Naffah et al., 2020; Sheldon et al., 2021; Vera Cala et al., 2020). De esta manera, distinguen a los estudiantes como: a) Adaptados al contexto, aquellos que se adaptaron al entorno universitario, pero no tienen un buen rendimiento; b) Adaptados a partir del rendimiento, aquellos que se adaptan a la Universidad porque les va bien, pero no tanto en términos de su entorno; c) Retenidos que no se han adaptado, aquellos que insisten en hacer parte de la Institución, pero en cualquier momento pueden desertar; y d) Desertores, aquellos que se fueron.

Como se puede observar, el acercamiento metodológico cualitativo ha permitido, por un lado, comprender la incorporación de los estudiantes a la Universidad y, por otro, develar tipologías estudiantiles que emergen a partir de las trayectorias de los jóvenes. Pero, al margen de esto, dichas tipologías dependen más de las características de los estudiantes, y de su proyecto de vida (Leal García et al., 2018), que de su relación con la conformación de los currículums; por tanto, se puede determinar que existe escasez de investigaciones que identifiquen tipologías estudiantiles desde la experiencia de quienes se enfrentan al currículum universitario y han decidido permanecer o abandonar, más aún en el contexto latinoamericano, y por cierto chileno, que es hacia donde apunta el desarrollo del presente estudio, y desde donde se ponen en consideración y discusión algunos aspectos representativos expresados a lo largo del documento.

\section{Metodología}

Orientado por los trabajos de Coulon (2017) y Dubet (2018) se utilizó una aproximación metodológica cualitativa-interpretativa, apoyada en la teoría comprensiva. De esta manera, el currículum universitario se definió en función de los siguientes elementos: planificación de clases, 
métodos de enseñanza-aprendizaje, evaluación del aprendizaje y uso de tecnologías de información y comunicación -TIC-.

En este sentido, las interacciones de los estudiantes con dichos elementos permitieron poner el foco en la idea de trayectoria estudiantil implícita en estos autores. Por lo que, en consecuencia, los análisis efectuados situaron su atención en la experiencia vivida por los jóvenes desde que ingresan a la Universidad hasta que optan por permanecer o abandonar. De esta manera, la investigación se llevó a cabo al año siguiente de haber ingresado a la Universidad, después de haber cursado a lo menos un semestre académico y cuando ya se dispone de información sobre permanencia y abandono.

En relación con los aspectos muestrales se deben tener en cuenta las siguientes consideraciones: preliminarmente a los hallazgos cualitativos presentados, el estudio desarrolló dos fases cuantitativas. La primera consideró un censo poblacional institucional que analizó información de 1454 estudiantes (359 que abandonaron y 1095 que permanecieron); y la segunda tuvo en cuenta la aplicación de un cuestionario en línea a 171 estudiantes (65 que abandonaron y 106 que permanecieron).

La Figura 1 presenta un esquema sintetizado del modelo metodológico empleado.

\section{Figura 1}

Síntesis del modelo metodológico

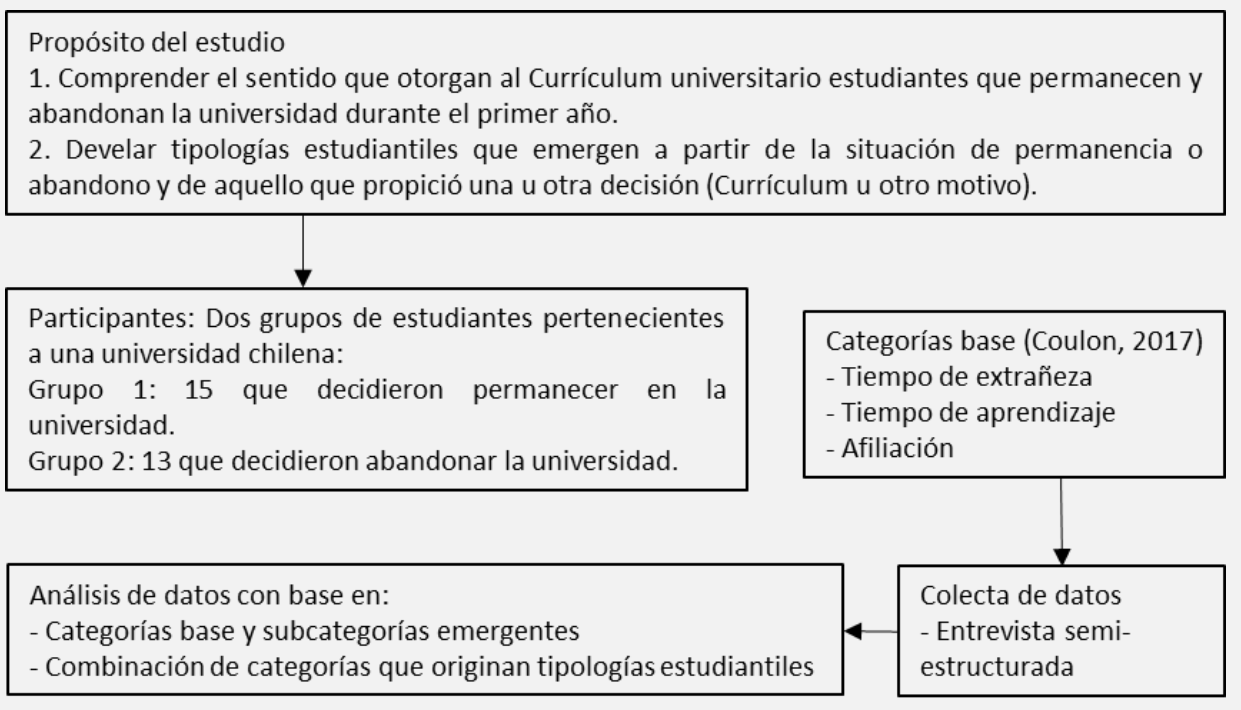

Nota: Elaboración de los autores. 
Quienes completaron el cuestionario en línea fueron invitados a hacer parte voluntariamente (luego de haber manifestado su consentimiento) de una entrevista semiestructurada, basada en las categorías de Coulon (2017), conformándose de esta manera dos grupos (13 que abandonaron y 15 que permanecieron), y estableciéndose cuatro (4) tipologías estudiantiles, asociadas al currículum y al proyecto de vida.

Posteriormente, con el apoyo del software de administración de datos cualitativos $Q D A$ Miner se generó una revisión preliminar de las entrevistas efectuadas a los dos grupos de estudiantes. Así, surgieron subcategorías para cada una de las categorías de base propuestas. La Tabla 1 muestra dichas subcategorías, las cuales fueron clasificadas dependiendo de la situación de permanencia o abandono expuesta, lo que permitió acceder a una comprensión más precisa de la trayectoria de los jóvenes, previa a su decisión. De acuerdo con la orientación evidenciada en dichas respuestas se codificó cada subcategoría con un signo (+) o (-) dependiendo del sentido de la favorabilidad de esta.

\section{Tabla 1}

Árbol de categorías

\begin{tabular}{|c|c|c|}
\hline Categorías/Subcategorías & Permanecen & Abandonan \\
\hline $\begin{array}{l}\text { Tiempo de extrañeza: } \\
\text { Ruptura entre la educación secundaria y la } \\
\text { inserción en la educación universitaria. }\end{array}$ & $\begin{array}{l}\text { Ruptura entre } \\
\text { niveles (-) } \\
\text { Elementos del } \\
\text { currículum (-) } \\
\text { Valoración de } \\
\text { estrategias (+) } \\
\text { Uso de TIC (+) }\end{array}$ & $\begin{array}{c}\text { Ruptura entre niveles (-) } \\
\text { Percepción de malas } \\
\text { bases académicas (-) } \\
\text { Elementos del } \\
\text { currículum (-) } \\
\text { Demandas al currículum } \\
(-) \\
\text { Uso de TIC (-) }\end{array}$ \\
\hline $\begin{array}{l}\text { Tiempo de aprendizaje: } \\
\text { Ambigüedad entre el anterior y el nuevo } \\
\text { escenario. }\end{array}$ & $\begin{array}{l}\text { Adaptación en el } \\
\text { proceso }(+) \\
\text { Facilitadores del } \\
\text { currículum }(+)\end{array}$ & $\begin{array}{l}\text { Desaprendizaje (-) } \\
\text { Obstaculizadores del } \\
\text { currículum (-) }\end{array}$ \\
\hline $\begin{array}{l}\text { Tiempo de afiliación: } \\
\text { Adaptación a la educación universitaria. }\end{array}$ & $\begin{array}{l}\text { Momento de la } \\
\text { adaptación }(+) \\
\text { Motivo } \\
\text { currículum }(+) \\
\text { Motivo proyecto } \\
\text { de vida }(+) \\
\end{array}$ & $\begin{array}{c}\text { Momento de la } \\
\text { desadaptación (-) } \\
\text { Motivo currículum (-) } \\
\text { Motivo proyecto de vida } \\
(-)\end{array}$ \\
\hline
\end{tabular}

Nota: Elaboración de los autores. 
La categoría Tiempo de extrañeza se da al ingreso del proceso formativo y es cuando los estudiantes reconocen las debilidades que arrastran desde la educación secundaria. La categoría Tiempo de aprendizaje aparece durante el proceso universitario y plantea la resolución o perpetuidad de los inconvenientes detectados inicialmente. Y la categoría Tiempo de afiliación se da cuando el estudiante, como parte del cierre de su proceso formativo, decide permanecer o abandonar sus estudios.

Esta codificación propuso, entonces, una estructura relacional entre las subcategorías "Motivo currículum" y "Motivo proyecto de vida", en términos de su particular injerencia dicotómica $((+)$ o (-)) sobre la decisión de permanencia o abandono. Esta situación dio origen a una matriz de análisis compuesta por cuatro (4) cuadrantes, cada uno de ellos en función de las categorías y subcategorías asociadas a las posibles combinaciones de las variables. Igualmente, esto posibilitó definir cuatro (4) tipologías de estudiantes (Tabla 2), en relación con la experiencia de los educandos con el currículum o su proyecto de vida, durante su primer año.

\section{Tabla 2}

Conformación de Tipologías Estudiantiles

\begin{tabular}{cll} 
& \multicolumn{1}{c}{ Motivo Currículum } & \multicolumn{1}{c}{ Motivo Proyecto de Vida } \\
\hline Permanecen & Tipo 1. Efectivos & Tipo 2. Con proyecto \\
\hline Abandonan & Tipo 4. Frustrados & Tipo 3. Sin proyecto \\
\hline
\end{tabular}

Nota: Elaboración de los autores.

\section{Resultados}

Los resultados proporcionaron, en primer lugar, la descripción del proceso que vivencian los estudiantes, según los tres momentos considerados por Coulon (2017), desde que ingresan a la Universidad hasta cuando deciden permanecer o abandonar. Posteriormente, se ofreció una clasificación de cuatro (4) tipologías de estudiantes, la cual surgió a partir de una estructura de relaciones, según las categorías y subcategorías de análisis definidas. 


\section{Sentido asignado al currículum por estudiantes que permanecen y abandonan}

\section{Categoría "Tiempo de extrañeza" (Al ingreso)}

El ingreso de los educandos a la Universidad evidenció una ruptura entre el nivel secundario y el universitario. En este sentido, ambos grupos (permanecen y abandonan) reconocieron en el ambiente universitario un mayor nivel de exigencia y una docencia más despersonalizada, alejada del contexto paternalista de la educación secundaria; condiciones que señalaron diferencias importantes entre ambos entornos formativos, las cuales inciden en el momento de enfrentar una educación superior.

Estas discrepancias fueron más evidentes en quienes abandonan que en quienes permanecen, pues el primer grupo consideró que llegó a la Universidad con considerables falencias en el dominio de los contenidos, puesto que la educación recibida en secundaria no le ayudó a integrarse académicamente a la Universidad; lo que, sumado al nivel de exigencia, generó las primeras inquietudes en torno a la continuación de sus estudios. En este sentido, esta población demandó a la institución un sistema de nivelación, que compensara las carencias académicas que se arrastran desde la educación secundaria (Martín Alonso et al., 2018).

Por otro lado, los dos grupos reconocieron las dificultades iniciales al enfrentarse al currículum de sus carreras, las cuales subsistieron en el grupo que abandona. Dificultades que existieron producto de las brechas mencionadas, y que para dicho grupo no se subsanaron con la labor docente, pues exigieron de sus formadores y de su institución una mayor preocupación. Esta percepción no fue tan radical para el grupo que permanece.

En lo que sí coincidieron ambos grupos fue en que las clases necesitaban una mayor estructuración y rigor, especialmente en la especificidad temática y las evaluaciones. En cuanto a lo primero criticaron la cantidad de temas, y en relación con lo segundo su alta exigencia y complejidad. Respecto a la metodología de enseñanza, los dos grupos valoraron el trabajo colaborativo, aunque demandaron una mayor comunicación docente, especialmente cuando se hizo parte de un grupo numeroso.

Por último, el uso de las TIC también evidenció significativas diferencias entre los dos grupos, en tanto, para los que permanecen, estas fueron vistas como herramientas que 
verdaderamente apoyaron los procesos de aprendizaje; mientras que para quienes abandonan, estas fueron instrumentos que dificultaron la formación, al considerarlas elementos complejos que demandaron una inducción adicional y un mayor tiempo de dedicación (especialmente en el uso de la plataforma institucional).

\section{Categoría "Tiempo de aprendizaje" (Durante el proceso)}

En el transcurso del proceso educativo los estudiantes que permanecen fueron superando algunas de las situaciones problema, evidenciadas en la categoría "Tiempo de extrañeza", a partir de la adquisición o transformación de sus hábitos académicos: mayor organización de sus tiempos, estudio sistemático, mayor responsabilidad, modificación de sus técnicas de estudio, adaptación a las metodologías, comunicación con su profesor, planificación de horarios, autonomía; reconociendo de esta forma que sus prácticas exitosas de secundaria ya no fueron suficientes.

Por su parte, para los estudiantes que abandonan esta adaptación fue más compleja o no existió, por lo que sintieron una especie de desaprendizaje que les impidió avanzar al ritmo de sus compañeros, provocando desconcierto, frustración y sensación de rezago, acrecentando su problemática situación de ingreso. Así, sus expectativas iniciales dejaron de coincidir con lo que les ofreció el desarrollo del proceso.

De esta manera, el currículum como elemento facilitador del proceso fue percibido solo por los estudiantes que permanecen, quienes consideraron que el apoyo metodológico y formativo favoreció su aprendizaje; mientras que para los que abandonan, su sentir desmotivado los llevó a observar mayores obstáculos y tensiones en su formación, especialmente entre lo que se trabajó en clase y lo que se evaluó.

\section{Categoría "Tiempo de afiliación" (Permanencia o abandono)}

La decisión final acerca de la permanencia o abandono de un estudiante se tomó a partir de lo expuesto y de que quienes permanecen consideraron que empezaron a obtener avances o logros en su trayectoria académica, situación que les motivó a continuar luego de su primer año de estudios. Por el contrario, quienes abandonan comenzaron a gestar dicha decisión a los pocos 
meses de haber ingresado, pues empezaron a obtener evaluaciones deficientes, a reprobar cursos y a percibir que se fueron quedando rezagados.

Respecto a los elementos del currículum que incidieron en esta decisión, también aparecieron diferencias significativas. Quienes decidieron permanecer consideraron que las TIC favorecieron el aprendizaje y complementaron el proceso, posición contraria a quienes abandonan, quienes las vieron como un impedimento. En cuanto a la metodología y las evaluaciones, ambos grupos las sintieron complejas al comienzo, pero luego quienes permanecen reconocieron su aporte sustantivo a la formación, mientras que quienes abandonan las consideraron complejas todo el tiempo. Y en cuanto a la concepción de la labor del profesor, esta fue considerada válida e importante para quienes permanecen, no tanto para quienes abandonan.

Adicional a lo anterior, el grupo que permanece presentó indicios de tener un proyecto de vida claro a futuro y sintió que la Universidad pudo contribuir a ello, razón por la cual estos estudiantes fueron superando los escollos, adaptándose a la institución. Lo que no ocurrió tan evidente para el grupo que abandona, el cual pareció transitar por la Universidad por el simple cumplimiento de un rol social, carente de articulación con un proyecto de vida, situación que enfrentó sus experiencias académicas con sus expectativas de vida (Hartwell et al., 2019).

\section{Tipologías estudiantiles}

En la Tabla 3 se exponen las tipologías estudiantiles construidas, las cuales surgieron de la combinación de las subcategorías "Motivo currículum" y "Motivo proyecto de vida", con las situaciones de permanencia o abandono. 


\section{Tabla 3}

Tipologías estudiantiles

\begin{tabular}{|c|c|c|}
\hline & Motivo Currículum & Motivo Proyecto de Vida \\
\hline \multirow[b]{2}{*}{ 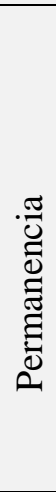 } & $\begin{array}{l}\text { Tipo } 1 \text {. Efectivos } \\
\text { Percibieron al currículum como un facilitador } \\
\text { del proceso formativo, permaneciendo por su } \\
\text { incidencia. }\end{array}$ & $\begin{array}{l}\text { Tipo 2. Con proyecto } \\
\text { Percibieron al currículum como un } \\
\text { facilitador del proceso formativo, } \\
\text { permaneciendo gracias a la presencia de un } \\
\text { proyecto de vida. }\end{array}$ \\
\hline & $\begin{array}{l}\text { Estudiantes que reconocieron las dificultades } \\
\text { iniciales al enfrentarse al currículum } \\
\text { universitario, pero que lograron adaptarse y } \\
\text { reconocer al currículum como un facilitador de su } \\
\text { avance académico, situación que los indujo a } \\
\text { permanecer. }\end{array}$ & $\begin{array}{l}\text { Estudiantes que reconocieron las } \\
\text { dificultades iniciales al enfrentarse al } \\
\text { currículum universitario; sin embargo, } \\
\text { durante el proceso tendieron a valorarlo, } \\
\text { decidiendo permanecer, principalmente } \\
\text { debido a que poseían metas definidas. }\end{array}$ \\
\hline \multirow[b]{2}{*}{ 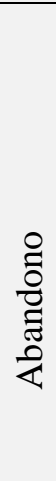 } & $\begin{array}{l}\text { Tipo 4. Frustrados } \\
\text { Percibieron al currículum como un obstáculo } \\
\text { del proceso formativo, abandonando por su } \\
\text { incidencia. }\end{array}$ & $\begin{array}{l}\text { Tipo 3. Sin proyecto } \\
\text { Percibieron al currículum como un } \\
\text { obstáculo del proceso formativo y } \\
\text { decidieron abandonar debido a que no } \\
\text { contaron con un proyecto de vida claro. }\end{array}$ \\
\hline & $\begin{array}{l}\text { Estudiantes que no contaron con bases } \\
\text { académicas importantes y que no las adquirieron } \\
\text { en su formación universitaria, considerando al } \\
\text { currículum como la razón por la que se rezagaron } \\
\text { y no lograron aprender, situación que los llevó a } \\
\text { no adaptarse y, finalmente, a abandonar. }\end{array}$ & $\begin{array}{l}\text { Estudiantes que no contaron con bases } \\
\text { académicas importantes y que no las } \\
\text { adquirieron en su formación universitaria, } \\
\text { asumiendo el currículum como un } \\
\text { sinsentido que no les permitió obtener } \\
\text { buenos resultados. }\end{array}$ \\
\hline
\end{tabular}

Nota: Elaboración de los autores.

Ahora, se explican a profundidad cada una de ellas.

\section{Tipología 1. Estudiantes "Efectivos"}

Conglomerado que reunió a aquellos estudiantes que permanecieron seducidos por el currículum de sus carreras. Si bien, como las demás tipologías, reconocieron las dificultades iniciales de adaptación, fueron capaces de sobrellevarlas, justificando así su decisión de permanencia.

Algunas de sus opiniones, vertidas en las entrevistas semiestructuradas, fueron: "la adaptación a la Universidad no es fácil, los profesores tenían una mayor preparación y son más exigentes que en el colegio", "tuve que tener personalidad, dejar la pena a un lado, mejorar mi 
comunicación, especialmente con gente mayor que yo", "la metodología de los profesores es súper buena, al igual que su calidad, no puedo dejar de estudiar por pequeños problemas que surgen, como hacen otros compañeros", "debo aprovechar que la Universidad me abrió sus puertas, debo tener convicción acerca de mí mismo", "conocer a diferentes tipos de personas es muy importante, cambiar mis grupos de trabajo".

Lo anterior, mostró que, si bien los estudiantes percibieron cambios sal ingresar a la Universidad, estos fueron vistos como desafíos y sorteados sin mayores contratiempos, siendo el currículum uno de los aspectos más novedosos para ellos, el cual fue incorporado efectivamente en sus vidas académicas y a su plan de vida. De esta manera, ellos se sintieron más próximos a la Institución y respaldados por una planificación seria.

\section{Tipología 2. Estudiantes "Con proyecto"}

La segunda tipología fue la de estudiantes "Con proyecto", la cual representó a quienes permanecieron en la Universidad debido a motivos ajenos al currículum, pero asociados a un proyecto de vida claro.

Entre sus principales expresiones se contaron: "me gusta que la Universidad se enfoque en desarrollar mi pensamiento crítico, que me pregunte sobre el presente y futuro de mi vida", "el liceo no nos permitía mucha libertad de pensamiento, pues el profesor daba todas las respuestas y el alumno, simplemente, reproducía la información, así que en ese escenario no había mucho qué planificar de la vida. Esta posibilidad fue algo que me impresionó al ingresar a la Universidad", "lo que era suficiente para el colegio, no lo es para la Universidad, aquí debo dedicar más tiempo a las actividades, debo ser más organizado como persona", "me gusta que los profesores sean variados, que sus formas de hacer sean distintas, así aprendo más y puedo, incluso, sugerir nuevas alternativas", "me siento motivado de solo pensar en que puedo construir mi vida", "quedarme fue mi decisión, pues solo así puedo realizarme como persona. Quiero ser profesora y por ello voy a terminar mi carrera".

Con esto se pudo constatar que este grupo de estudiantes reconoció las dificultades existentes en el nuevo proceso formativo, pero percibió que los esfuerzos desplegados por la Universidad, para posibilitar su éxito académico y permitir el desarrollo de su proyecto de vida, 
fueron muy superiores a las vicisitudes. Por tanto, al superar los inconvenientes iniciales, su aspiración personal, sus ganas de salir adelante y su convicción de proyectarse desde la profesión, fueron suficientes para orientar su decisión acerca de quedarse en la Universidad.

\section{Tipología 3. Estudiantes "Sin proyecto"}

Estos estudiantes advirtieron dificultades desde su ingreso a la Universidad, puesto que no tuvieron claros sus objetivos de vida o de formación. Adicionalmente, tuvieron una precaria percepción acerca de sus raíces formativas, lo que les dificultó mucho más su posibilidad de enfrentar los estudios universitarios. Así mismo, dichas falencias se acrecentaron conforme pasó el tiempo, lo que les generó frustración y, posteriormente, todo esto los condujo a la deserción.

En este contexto, algunas de sus opiniones fueron: "los profesores no me entienden, no comprenden mi pensamiento de vida", "la Universidad no me colabora. Pareciera que mis metas no son coherentes con lo que ellos pretenden", "carecer de una planificación clara de mi parte hace difícil mi proceso académico", "en la Universidad debo estudiar más de lo que yo creí. No es suficiente con mantener mis tiempos de colegio", "es muy frustrante estudiar y estudiar y que aun así me vaya mal", "creo que mis gustos nos son consecuentes con lo que veo en clase", "he llegado al punto de preguntarme: ¿qué quiero hacer con mi vida?”.

\section{Tipología 4. Estudiantes "Frustrados"}

Esta última tipología aglutinó a aquellos estudiantes carentes de un claro proyecto de vida y de una adaptación a las exigencias propuestas por el currículum universitario. Estos alumnos "vivieron frustrados" al no poder superar con éxito los desafíos de su nuevo estatus académico, situación que se agravó a medida que pasó el tiempo, lo que repercutió en su motivación por estudiar y por participar en los ejercicios universitarios. Incluso, en este grupo existieron estudiantes que abandonaron desde muy temprano su carrera.

Al respecto, comentaron: "es que en la Universidad todo cambia. Es mucho más exigente que el colegio y los cursos son más difíciles", "en la Universidad se debe tener más tiempo para estudiar", "la metodología de mi carrera me exige participar y a mí eso me da como pena, así que 
yo solo voy a que me vean y ya", "después del primer nivel, las evaluaciones se hicieron más difíciles. Al final ya ni las quería presentar", "eso fue como en cadena, una primera nota mala y luego todas las demás".

Es así como, luego de tipificar a los estudiantes, la Figura 2 presenta las opiniones de ambos grupos (permanencia y abandono) en cuanto a la relevancia del currículum en su decisión, evidenciando que ambos conglomerados tuvieron experiencias distintas, y hasta contrarias en su percepción. El $80 \%$ de los encuestados que permanecen consideró que el currículum fue importante o muy importante en su decisión, mientras que para quienes abandonan, el 76,5\% determinó que este mismo elemento fue un factor importante o muy importante, pero para dejar la Universidad.

\section{Figura 2}

Importancia del currículum en la decisión de permanecer o abandonar

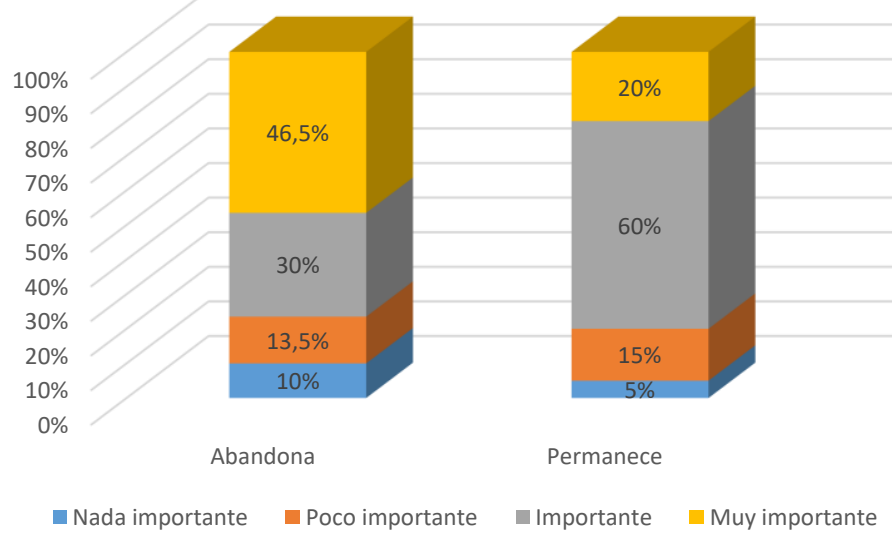

Nota: Elaboración de los autores.

Por otro lado, en cuanto a la relevancia del proyecto de vida, la Figura 3 muestra que para quienes decidieron permanecer, la posibilidad de tener y lograr un proyecto de vida, contando con el aporte de la Universidad, fue importante o muy importante en un $95 \%$; a diferencia de lo que sucedió con quienes abandonan, donde existió un amplio espectro de opiniones acerca de no encontrar que esto fuera un aspecto crucial para su permanencia (40\%). 


\section{Figura 3}

Importancia del proyecto de vida en la decisión de permanecer o abandonar

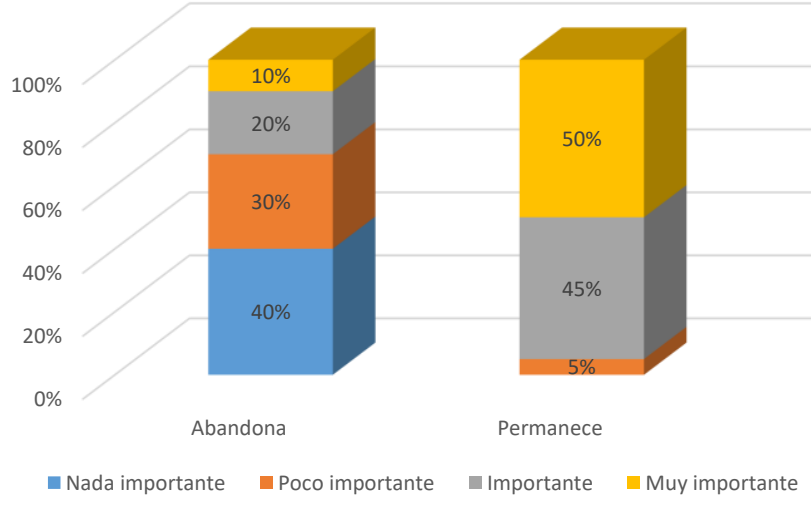

Nota: Elaboración de los autores.

Como quedó en evidencia, en términos generales, quienes permanecen en la Universidad encontraron una relación importante entre el currículum de su carrera, su proyecto de vida y su decisión (Guitart, 2019). Caso contrario ocurrió con quienes decidieron desertar. Esto demuestra que un factor importante en dicha ruta formativa estuvo condicionado por el interés y la intención del estudiante (Becker et al., 2019), así como por su comprensión acerca de la función de la Universidad y su contribución a la edificación de su proyecto de desarrollo personal.

\section{Discusión}

Como aporte a la revisión de la literatura presentada, relacionada con la permanencia y deserción de los estudiantes en sus estudios superiores y sus distintas clasificaciones, la contribución del presente artículo está dada en señalar que existen múltiples causas que condicionan la posibilidad de abandono, como bien mencionan autores como Berka \& Marek (2021); sin embargo, el currículum juega un papel fundamental en incrementar esta situación proclive a los estudiantes. Por ello, en general, se puede configurar esta relación abandono / no abandono desde la perspectiva de tres elementos que coexisten y se relacionan con las expectativas asociadas al mismo proyecto de vida. Estos tres elementos se relacionan estrictamente con los hitos o momentos de tránsito del estudiante en la vida universitaria (donde lo cuantitativo, como bien 
expresan Tinto (1975) y demás autores de la corriente funcionalista norteamericana, tiene una incidencia fundamental).

Como primer punto, aparecen las condiciones de ingreso, momento en el cual los estudiantes tienen muchas expectativas con respecto al futuro (Bäulke et al., 2021), y la identificación de un proyecto de vida futuro a partir de la preparación académica se vuelve determinante para generar motivación por el proceso de aprendizaje. En la medida que existe una mayor orientación sobre los campos disciplinares de la formación a recibir, se minimiza la transferencia inter-programas o incluso la deserción de la educación superior. Por ello, en el ingreso es fundamental incorporar procesos de acompañamiento académico, pero también emocional, para garantizar la pertinencia en la formación y también la motivación en términos de un proyecto de vida (instancia en la que los aspectos cualitativos, señalados por Coulon (2017) y Dubet (2018) desde la sociología francesa, tienen una incidencia crucial).

En un segundo momento, aparece la permanencia en el transcurso de la formación. Aquí, la posibilidad de abandono es mucho más alta en los primeros cuatro semestres que en el resto, como bien expresa la literatura sobre la temática (Castro Montoya, Manrique Hernández et al., 2020). Por tanto, las medidas fundamentales se concentran en garantizar una articulación y seguimiento de los indicadores de desempeño en el nivel curricular del programa, así como a nivel individual. El monitoreo y seguimiento sobre el desempeño de los estudiantes, las estadísticas de evaluación curricular y los aspectos de fortalecimiento en formación integral se vuelven importantes para expresar una postura institucional en la minimización de la posibilidad de abandono.

El tercer hito aparece al final, y se refiere a las condiciones de egreso. Aunque la posibilidad de abandono es inferior en esta etapa, es posible que asignaturas de corte profesional involucren un manejo teórico práctico que pondría el acento en la praxis, lo que se convertiría en una confrontación final sobre el destino profesional del estudiante (Castro Montoya, Lopera Gómez et al., 2021). En la misma medida, los trabajos culminantes de grado suelen tener un nivel de exigencia que se refleja en tiempos y recursos que deben ser destinados para culminar con éxito el ejercicio monográfico. En algunas ocasiones esto puede causar una diferencia entre el concepto de egresado (culminación de todas las asignaturas de un plan de estudios) y el concepto de graduado (aquel que recibe su diploma una vez culminado el respectivo trabajo de grado). 
En este orden de ideas, el concepto de abandono que aquí se esboza es coincidente con buena parte de los estudios consultados, adjudicando un peso preponderante al papel del plan de estudios y al proyecto vida en el abordaje de las expectativas de los estudiantes, situación que posibilita hablar de las tipologías definidas, las cuales tienen diversos niveles de relación con las propuestas de Soler Julve (2014), Pineda Lezama y Alcántara Galdámez (2017), Hurtado Bello et al. (2017) y Jenert et al. (2017), pero tienen su propio valor a partir de la metodología empleada y los hallazgos encontrados en el presente estudio.

\section{Conclusiones}

Una primera conclusión del estudio es la clara identificación de la existencia del proceso que vive el estudiante, previamente a tomar la decisión de permanecer o desertar en sus estudios de Universidad. La ruptura evidenciada por el estudiante, al enfrentarse al currículum universitario, constituye la fase de extrañeza identificada por Coulon, la cual en algunos casos es definitivamente impactante para los jóvenes, mucho más para quienes desde un comienzo se perciben con una base formativa deficiente y no encuentran en la Universidad una clara solución.

De esta manera, se distinguen los primeros desajustes entre la educación secundaria y la educación universitaria, lo que se agudiza cuando no se favorece el tránsito entre los dos niveles; condición que evidencia una importante falencia en el sistema educativo, en este caso chileno, que no es ajena al resto del continente. Además, esta situación desfavorece la permanencia del estudiante y promueve su temprano abandono.

A partir de esto, las tipologías estudiantiles develadas permiten identificar perfiles comunes de estudiantes, según su condición de permanencia o abandono, caracterizándolos y encontrando elementos que, de alguna manera, pueden ayudar a proponer soluciones a la problemática evidenciada o tejer puentes entre los dos niveles de formación, con la intención de combatir la deserción.

Una de estas soluciones puede ser la concientización en el estudiante acerca de la importancia de tener en cuenta la relación entre su desempeño académico, el currículum propuesto por la Universidad y su proyecto de vida, siendo esta una articulación necesaria para acrecentar su sentido de permanencia, ya que de esta forma puede entender que la Universidad puede llegar a 
ser un escenario aliado de su propio desarrollo, al servicio de sus metas y objetivos. Solución que también implica una atención por parte de la Universidad, ya que si esta quiere combatir el abandono, debe comprender que sus currículums deben encantar al estudiante, particularmente a aquellos que no están muy seguros de su proyecto de vida; por lo que la institución debe proponer currículums más flexibles, que ofrezcan diversas trayectorias formativas, que articulen las diversas carreras y que le muestren un panorama mucho más amplio al estudiante, en el que tenga la posibilidad de experimentar una evolución en su desarrollo personal y académico, tanto de manera individual como colectiva.

Adicional a esto, queda la sensación de que los jóvenes visualizan el primer año de estudios universitarios como una especie de "prueba" que pueden o no superar, lo que deja en evidencia una concepción inmediatista del estudiante, particularmente de quienes no poseen un claro proyecto de vida. Y esto se agrava cuando la institución no crea, durante ese primer año, estrategias sistémicas, claras y eficientes en pro de la permanencia, que "amortigüen" el paso de un nivel formativo a otro y que incentiven en el educando una preocupación mucho más profunda por su propio bienestar futuro.

Finalmente, se concluye que la decisión de permanencia o abandono exige avanzar en propuestas curriculares coherentes, en permanente evaluación y retroalimentación, incluyendo la de los propios agentes educativos, donde las acciones que se emprendan requieren estar en sintonía con la inducción asertiva a la vida universitaria, en sincronía con el currículum y el plan de vida, y no implementarse de manera fortuita o desvinculada, puesto que queda demostrado que las experiencias de aula, vividas por el estudiante, afectan la decisión de su trayectoria formativa.

\section{Referencias}

Asociación Colombiana de Universidades. (25-26 de septiembre de 2018). Tasa de deserción estudiantil. II Foro Internacional de Educación y Movilidad Social, Barranquilla, Colombia.

Bäulke, L., Grunschel, C., \& Dresel, M. (2021). Student dropout at university: A phase-orientated view on quitting studies and changing majors [Abandono de los estudiantes en la universidad: una visión por fases sobre el abandono de los estudios y el cambio de carrera]. 
European Journal of Psychology of Education: A Journal of Education and Development, 1-24. https://doi.org/10.1007/s10212-021-00557-x

Bean, J., \& Metzner, B. (1985). A conceptual model of nontraditional undergraduate students' attrition [Un modelo conceptual de deserción de estudiantes universitarios no tradicionales]. Review of Educational Research, 55(4), 485-540. https://doi.org/10.2307/1170245

Becker, S., Pfost, M., Schiefer, I. M., \& Artelt, C. (2019). Relations between life-goal regulation, goal orientation, and education-related parenting - A person-centered perspective [Relaciones entre la regulación de las metas de vida, la orientación de las metas y la paternidad relacionada con la educación: una perspectiva centrada en la persona]. Learning and Individual Differences, 76(1), 1-6. https://doi.org/10.1016/j.lindif.2019.101786

Berka, P., \& Marek, L. (2021). Bachelor's degree student dropouts: Who tend to stay and who tend to leave? [Desertores de estudiantes de licenciatura: ¿Quiénes tienden a quedarse y quiénes tienden a irse?] Studies in Educational Evaluation, 70. https://doi.org/10.1016/j.stueduc.2021.100999

Castrillón Gómez, O. D., Sarache, W., y Ruíz Herrera, S. (2020). Predicción de las principales variables que conllevan al abandono estudiantil por medio de técnicas de minería de datos. Formación Universitaria, 13(6), 217-228. https://doi.org/10.4067/S0718$\underline{50062020000600217}$

Castro Montoya, B. A., Lopera Gómez, C. M., Manrique Hernández, R. D., y González Gómez, D. (2021). Modelo de riesgos competitivos para deserción y graduación en estudiantes universitarios de programas de pregrado de una universidad privada de Medellín (Colombia). Formación Universitaria, 14(1), 81-98. https://doi.org/10.4067/S0718$\underline{50062021000100081}$

Castro Montoya, B. A., Manrique Hernández, R. D., González Gómez, D., y Segura Cardona, A. M. (2020). Trayectoria académica y factores asociados a graduación, deserción y rezago en estudiantes de programas de pregrado de una universidad privada de Medellín (Colombia). Formación Universitaria, 13(1), 43-54. https://doi.org/10.4067/S0718$\underline{50062020000100043}$ 
Chalela Naffah, S., Valencia Arias, A., Ruiz Rojas, G. A., y Cadavid Orrego, M. (2020). Factores psicosociales y familiares que influyen en la deserción en estudiantes universitarios en el contexto de los países en desarrollo. Revista Lasallista de Investigación, 17(1), 103-115. https://doi.org/10.22507/rli.v17n1a9

Christiane, D. (2019). Bologna - A utopy of harmony for European higher education [Bolonia: una utopía de armonía para la educación superior europea]. Innovation: The European Journal of Social Sciences, $\quad 32(4), \quad 403-405$. https://doi.org/10.1080/13511610.2019.1674131

Coulon, A. (2017). Le métier d'étudiant. L'entrée dans la vie universitaire [El trabajo de un estudiante. Entrada a la vida universitaria]. Educaçao e Pesquisa, 43(44), 1239-1250. https://doi.org/10.1590/s1517-9702201710167954

Dubet, F. (2018). Trois jeunesses. La révolte, la galère, l'émeute. Lormont: Le bord de l'eau [Tres jóvenes. La revuelta, la cocina, el motín. Lormont: el borde del agua]. Revista Colombiana de Ciencias Sociales, 10(1), 220-234. https://doi.org/10.21501/22161201.3064

European Commission. (2018). The European Higher Education Area in 2018: Bologna Process Implementation Report [El Espacio Europeo de Educación Superior en 2018: Informe de implementación del proceso de Bolonia]. European Union. https://op.europa.eu/en/publication-detail/-/publication/2fe152b6-5efe-11e8-ab9c01aa75ed71a1/languageen?WT.mc_id=Selectedpublications\&WT.ria_c=677\&WT.ria_f=706\&WT.ria_ev=search

Fonseca Grandón, G. (2018). Trayectorias de permanencia y abandono de estudios universitarios: una aproximación desde el currículum y otras variables predictoras. Educación y Educadores, 21(2), 239-256. https://doi.org/10.5294/edu.2018.21.2.4

Fonseca Grandón, G., y García, F. (2016). Permanencia y abandono de estudios en estudiantes universitarios: análisis desde la teoría organizacional. Revista de la Educación Superior, 45(179), 25-39. https://doi.org/10.1016/j.resu.2016.06.004

Gómez-Molina, S., Palacios-Moya, L., Berrio-Calle, J. E., Gaviria-Zapata, S., Quiceno-Merino, L. M., y Figueroa-Álvarez, P. (2019). Modelo de satisfacción de egresados universitarios: un estudio de caso. Revista CEA, 5(10), 49-68. https://doi.org/10.22430/24223182.1443 
Guitart, M. E. (2019). Identity in education and education in identities: Connecting curriculum and school practice to students' lives and identities [Identidad en la educación y educación en identidades: Conectando el currículo y la práctica escolar con las vidas e identidades de los estudiantes]. Springer International Publishing. https://doi.org/10.1007/978-3-030$\underline{28412-1 \_10}$

Hartwell, J. C., Upadhyay, Y., \& Sourani, A. (2019). Influential factors of life cycle management in education PFI projects [Factores que influyen en la gestión del ciclo de vida en proyectos PFI educativos]. Built Environment Project and Asset Management, 9(2), 302-314. https://doi.org/10.1108/BEPAM-02-2018-0043

Hurtado Bello, P., Tamez Almaguer, R., y Lozano Rodríguez, A. (2017). Características que presentan los estudiantes con estilos de aprendizaje diferentes en ambientes de aprendizaje $\begin{array}{llll}\text { colaborativo. } & \text { Tendencias } & \text { Pedagógicas, } & 30,\end{array}$ https://doi.org/10.15366/tp2017.30.011

Jenert, T., Brahm, T., Gommers, L., \& Kühner, P. (2017). How do they find their place? A typology of students' enculturation during the first year at a business school [¿Cómo encuentran su lugar? Una tipología de la inculturación de los estudiantes durante el primer año en una escuela de negocios]. Learning, Culture \& Social Interaction, 12, 87-99. https://doi.org/10.1016/j.lcsi.2016.12.001

Klees, S. J. (2017). Quantitative methods in comparative education and other disciplines: are they valid? Educação \& Realidade, 42(3), 841-858. https://doi.org/10.1590/2175-623664816

Köhler, T., Landis, R., \& Cortina, J. M. (2017). Establishing methodological rigor in quantitative management learning and education research: the role of design, statistical methods, and reporting standards [Establecer el rigor metodológico en el aprendizaje de la gestión cuantitativa y la investigación educativa: el papel del diseño, los métodos estadísticos y los estándares de presentación de informes.]. Academy of Management Learning \& Education, 16(2), 173-192. https://doi.org/10.5465/amle.2017.0079

Leal García, E., Martínez Neira, X., y Rodríguez Fernández, N. (2018). La cultura como vía para fortalecer la dimensión educativa del proceso de formación universitaria. EDUMECENTRO, 10(1), 229-235. http://scielo.sld.cu/pdf/edu/v10n1/edu16118.pdf 
Martín Alonso, D., Blanco García, N., y Sierra Nieto, J. E. (2018). Indagación narrativa sobre las tensiones vividas por un profesor de educación secundaria en el proceso de creación curricular. Education Policy Analysis Archives, 26(143/144), 1-22. https://doi.org/10.14507/epaa.26.3571

Modena, F., Rettore, E., \& Tanzi, G. M. (2020). The effect of grants on university dropout rates: evidence from the Italian case [El efecto de las becas en las tasas de abandono universitario: evidencia del caso italiano]. Journal of Human Capital, 14(3), 343-370. https://doi.org/10.1086/710220

Nurie, Y. (2019). Doctoral students research trends and practices: Implications for curriculum design in higher education [Tendencias y prácticas de investigación de estudiantes de doctorado: implicaciones para el diseño curricular en la educación superior]. College Quarterly, 22(2), 1-4. https://files.eric.ed.gov/fulltext/EJ1221334.pdf

Ñañez Silva, M. V., Pariona Luque, R. B., Quispe Calderón, J. C., \& Díaz Rengifo, H. (2021). Directive management and pedagogical innovation model for public universities [Modelo de gestión directiva e innovación pedagógica para universidades públicas]. 3C Empresa, 1, 33-45. https://doi.org/10.17993/3cemp.2021.specialissue1.33-45

Organización de las Naciones Unidas para la Educación, la Ciencia y la Cultura. (2015). La UNESCO y los Objetivos de Desarrollo Sostenible. UNESCO. https://es.unesco.org/sdgs

Pineda Lezama, O. B., y Alcántara Galdámez, N. J. (2017). Hábitos de estudio y rendimiento académico en estudiantes universitarios. Innovare. Ciencia y Tecnología, 6(2), 19-34. https://unitec.edu/innovare/published/volume-6/number-2/628-habitos-de-estudio-yrendimiento-academico-en-estudiantes-universitarios.pdf

Rodríguez Bustamante, A., Vicuña Romero, J. J., y Zapata Posada, J. J. (mayo-agosto, 2021). Familia y escuela: educación afectivo-sexual en las escuelas de familia. Revista Virtual Universidad Católica del Norte, (63), 312-344. https://www.doi.org/10.35575/rvucn.n63a12

Rodríguez Gómez, R. (2018). Dos décadas del Proceso de Bolonia. Revista Mexicana de Investigación Educativa, 23(76), 7-14. http://www.scielo.org.mx/pdf/rmie/v23n76/14056666-rmie-23-76-7.pdf 
Savela, T. (2017). The advantages and disadvantages of quantitative methods in schoolscape research. Studying the Visual and Material Dimensions of Education and Learning, Linguistics and Education,

44 ,

$31-44$. https://doi.org/10.1016/j.linged.2017.09.004

Seah, K. K. C., Pan, J., \& Tan, P. L. (2020). Breadth of university curriculum and labor market outcomes [Amplitud del currículo universitario y los resultados del mercado laboral]. Labour Economics, 65. https://doi.org/10.1016/j.labeco.2020.101873

Servicio de Información de Educación Superior. (2018). Informe de retención de $1^{e r}$ año de pregrado / cohortes 2013-2017. Ministerio de Educación del Gobierno de Chile. http://www.mifuturo.cl/wpcontent/uploads/2018/12/informe_de_retencion_sies_2018_02102018-1.pdf?x78460

Sheldon, E., Simmonds-Buckley, M., Bone, C., Mascarenhas, T., Chan, N., Wincott, M., Gleeson, H., Sow, K., Hind, D., \& Barkham, M. (2021). Prevalence and risk factors for mental health problems in university undergraduate students: A systematic review with metaanalysis [Prevalencia y factores de riesgo de problemas de salud mental en estudiantes universitarios: una revisión sistemática con metaanálisis]. Journal of Affective Disorders, 287, 282-292. https://doi.org/10.1016/j.jad.2021.03.054

Soler Julve, I. (2014). Una tipología de la población estudiantil universitaria. Revista de la Asociación de Sociología de la Educación, 7(1), 104-122. https://dialnet.unirioja.es/descarga/articulo/5144600.pdf

Tinto, V. (1975). Dropout from higher education: A theoretical synthesis of recent research. Review of Educational Research, 45(1), 89-125. https://doi.org/10.3102/00346543045001089

Vandelannote, I., \& Demanet, J. (2021). Unravelling socioeconomic school composition effects on higher education enrollment: the role of students' individual and shared feelings of futility and self-efficacy [Desentrañar los efectos de la composición socioeconómica de las escuelas en la matriculación en la educación superior: el papel de los sentimientos individuales y compartidos de inutilidad y autoeficacia de los estudiantes]. Social Psychology of Education, 1-25. https://doi.org/10.1007/s11218-021-09608-Z 
Velásquez Narváez, Y., y González Medina, M. A. (2017). Factores asociados a la permanencia de estudiantes universitarios: caso UAMM-UAT. Revista de la Educación Superior, 46(184), 117-138. https://doi.org/10.1016/j.resu.2017.11.003

Vera Cala, L. M., Niño García, J. A., Porras Saldarriaga, A. M., Durán Sandoval, J. N., Delgado Chávez, P. A., Caballero Badillo, M. C., y Navarro Rueda, J. P. (mayo-agosto, 2020). Salud mental y deserción en una población universitaria con bajo rendimiento académico. Revista Virtual Universidad Católica del Norte, (60), 137-158. https://www.doi.org/10.35575/rvucn.n60a8

Wild, S., \& Schoulze, L. (2020). Student dropout and retention: An event history analysis among students in cooperative higher education [Abandono y retención de estudiantes: un análisis del historial de eventos entre los estudiantes de la educación superior cooperative]. International Journal of Educational Research, 104. https://www.doi.org/10.1016/j.ijer.2020.101687 